\title{
Mesenchimal stem cells: a possible role in the pathogenesis and treatment of spondyloarthritis
}

\author{
F.M. Perrotta, G. Guerra, A. De Socio, S. Scriffignano, E. Lubrano \\ Department of Medicine and Health Sciences "Vincenzo Tiberio", University of Molise, Campobasso, Italy
}

\begin{abstract}
SUMMARY
Spondyloarthritis (SpAs) are a group of chronic inflammatory diseases that affect joints and enthesis with a possible involvement of other districts such as skin, eye and bowel. In SpAs, the inflammatory process could lead to both erosive damage (as in peripheral joint involvement of psoriatic arthritis), or bone formation (as in ankylosing spondylitis) with a reduction in function and quality of life. Recently, Mesenchimal stem cells (MSCs) transplant was used in different diseases, including autoimmune and inflammatory diseases, with the aim of repairing tissue damage, exploiting their regenerative capacity. However, MSCs also proved to have an immune-modulatory capacity due to their interaction with the cells of the immune system. The aim of this brief paper was to review the possible pathogenic role and the new perspective of MSCs use in SpAs.
\end{abstract}

Key words: Spondyloarthritis; Mesenchimal stem cells; Pathogenesis; Treatment.

\section{INTRODUCTION}

pondyloarthritis (SpAs) are a group of inflammatory diseases characterized by chronic inflammatory spondylitis, chronic synovitis and enthesitis associated with extra-articular manifestations (i.e., psoriasis, uveitis and inflammatory bowel disease). Traditionally, the term SpA comprises a group of interrelated yet distinguishable disorders $(1,2)$. These disorders are ankylosing spondylitis (AS), psoriatic arthritis (PsA), arthritis/SpA associated with ulcerative colitis or with Crohn's disease, reactive arthritis and undifferentiated SpA. All SpAs subgroups have in common a familial aggregation, and are associated to varying extents with human leukocyte antigenB27 (HLA-B27), providing evidence for a shared genetic background $(2,3)$. One of the most important aspects of SpAs was the involvement of bone and cartilage tissue: the development of inflammation at entheseal and synovial sites can lead to tissue damage that could be predominantly erosive, such as in PsA, or could be expressed as new bone formation such as in
AS. Several studies reported an occurrence of bone erosions ranging from 46 to $62 \%$ of patients affected by PsA, leading to joint destruction and severe reduction of function and quality of life (4). Likewise, the new bone formation with the fusion of the spine in the late stage could lead to a severe reduction of function and quality of life in AS (5). The introduction of biologic drugs, such as the tumor necrosis factor (TNF) blockers, the anti-interleukin (IL) 12/23 and anti-IL17 blockers for the treatment of SpAs, has been considered the greatest breakthrough over the past 50 years (6-10). However, their efficacy in slowing radiographic progression of the disease, in healing erosions or reversing bone formation is far from being proven. Since damaged and/or deformed joints cannot be repaired, a novel treatment strategy aimed at both anti-inflammation and bone regeneration/ healing is needed, especially in the long lasting form. In this context, the recent use in different clinical settings of mesenchimal stem cells (MSCs) could provide a possible new approach to treatment. In fact, it has been shown that MSCs are im-
Corresponding author

Ennio Lubrano

Department of Medicine

and Health Sciences "Vincenzo Tiberio", University of Molise

Via Giovanni Paolo II, C/da Tappino

86100 Campobasso, Italy

E-mail: enniolubrano@hotmail.com 
portant in the pathogenesis of many autoimmune diseases due to their proliferating and differentiating potential and because of their potent immune-modulatory and antiinflammatory properties (11). The aim of this paper was to review briefly the role of MSCs in the pathogenesis and treatment of $\mathrm{SpA}$ and to discuss the current border of cellular treatment in these diseases.

\section{MSCs: SOURCE AND IMMUNE-REGULATORY PROPERTIES}

\section{Source}

MSCs cells are found in a variety of tissues and have the ability to proliferate rapidly and differentiate into musculoskeletal lineages including bone, cartilage, muscle, marrow stroma and a variety of connec-

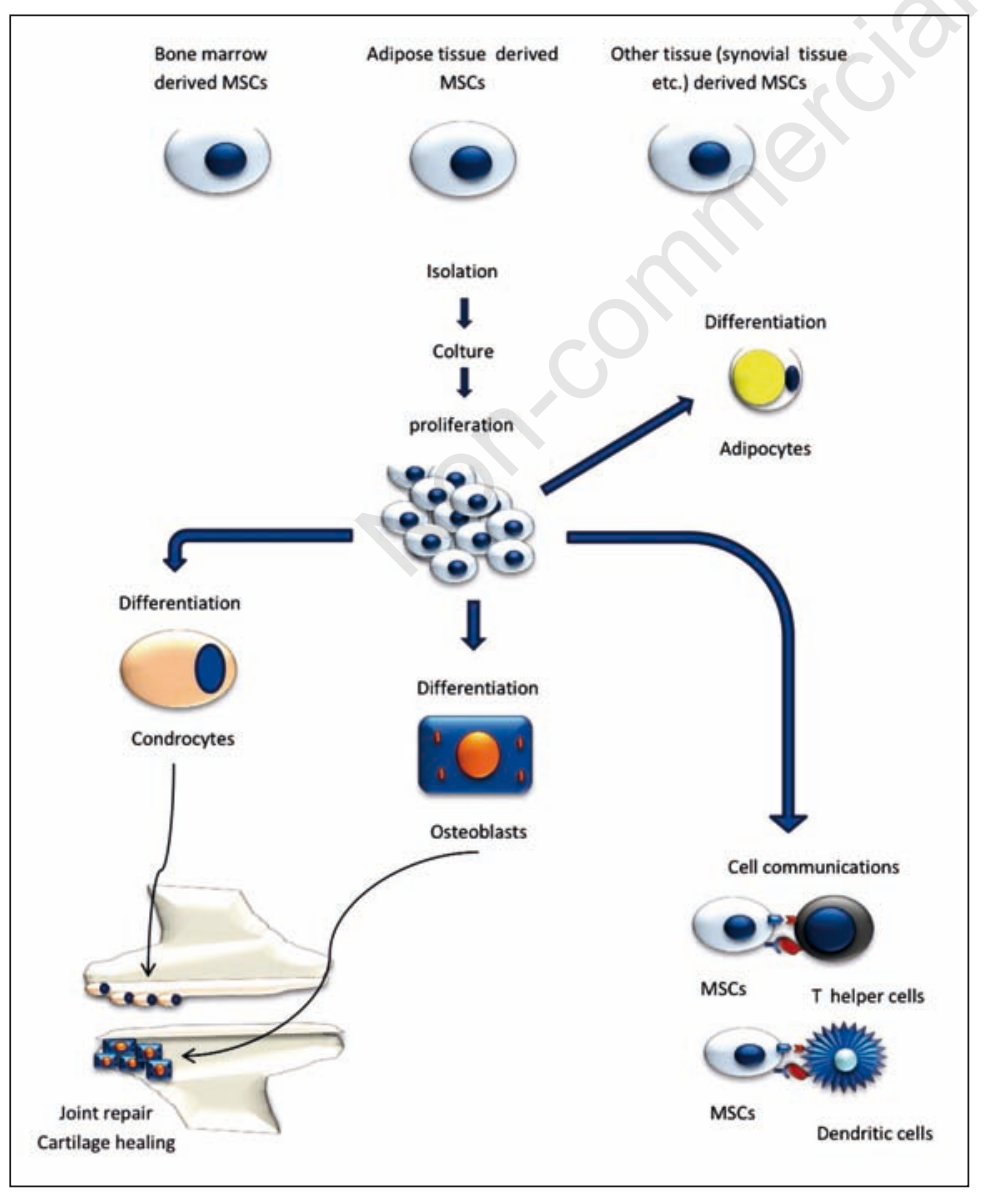

Figure 1 - Source and function of MSCs and their potential use in SpAs. tive tissues. The International Society for Cellular Therapy established the minimum criteria that must be met in order to classify a stem cell as MSCs: it must be:

1. adherent to plastic under standard culture conditions;

2. express CD105, CD73 and CD90;

3. lack expression of CD45, CD34, CD14 or CD11b, CD79a or CD19 and human leukocyte antigen (HLA)-DR;

4. differentiate from osteoblasts, adipocytes and chondroblasts in vitro (12).

MSCs tissue source is another area of active investigation: bone marrow, adipose tissue and umbilical cord blood have been most commonly used, but the wide distribution of these cells has generated interest in other locations such as synovium and periosteum $(13,14)$. Bone marrow-derived MSCs (BM-MSCs) are part of the haematopoietic niche, where they support haematopoiesis such as MSCs coming from other tissues supporting connective tissue homeostasis. The physiological role of MSCs is to ensure that when cells within tissues expire naturally, these can be easily replaced to provide physiological balance in the organism but also to serve as a reserve for damaged or compromised adult tissues requiring more extensive repair, regeneration or expansion. Curiously, the synovial membrane has proved to be a great source for the functionality of MSCs with highest multi-potentiality for adipogenesis, osteogenesis, chondrogenesis and myogenesis (15-17). Figure 1 summarizes the source and function of MSCs and their potential use in SpAs.

\section{Immunoregulation}

Different studies demonstrated that MSCs orchestrate important immunologic functions through modulation of the local inflammatory response (18), mainly in autoimmune diseases. It has been demonstrated that inflammation could alter the expression and function of MSCs; in fact, some studies showed that prevalence of MSCs was significantly lower in the synovial fluid of rheumatoid arthrtitis (RA) patients than in that of osteoarthritis patients (19, $20)$. In addition, there was a negative re- 
lationship between synovial MSCs' chondrogenic and clonogenic capacities and the magnitude of synovitis in RA patients (21), suggesting a suppression in MSCs' repair function within the joint, probably due to the high levels of inflammatory cytokines: TNF is indeed known to prevent the mesenchymal differentiation capabilities of MSCs in vitro $(22,23)$. Thus, in addition to the well-known catabolic effects of TNF on articular cartilage and bone, TNF signaling could decrease the reparative responses of endogenous joint MSCs, thereby limiting cartilage/bone regeneration during arthritis. The inhibition of structural damage progression in the joints of RA patients with anti-TNF therapy could be in part due to the re-activation of MSCs. Alongside their stem cell properties, culture-expanded MSCs have immune-modulatory properties. Studies predominantly using BM-MSCs have demonstrated that immunomodulation properties of MSCs are dependent on the secretion of cytokine and other factors including INF- $\gamma$ (24) and are largely mediated by factors such as indolamine 2,3-dioxygenase or nitric oxide synthase, resulting in inhibition of both $\mathrm{T}$ and B-cell proliferation and function (25). MSCs can also induce the differentiation of regulatory $\mathrm{T}$ cells (Treg) and maintain their inhibitory functions (26-28). Moreover, MSCs can also inhibit the differentiation of T cells in T helper 17 (Th17). Furthermore, MSCs suppress innate immunity through the inhibition of dendritic cell formation and function (29), by decreasing the expression of human leukocyte antigen DR, CD80 and CD86 co-stimulatory molecules on antigen presenting cells (29), and through the inhibitory effects on proliferation of both resting and IL-2 activated natural killer (NK) cells.

Moreover, a direct inhibition on the cytotoxic capabilities of NK cells (30) has been demonstrated. The immune-regulatory properties of cultured synovial MSCs are less well known but the data available seems to show that they have similar functions in respect to BM-MSCs $(31,32)$. This immune-regulatory potential could be important in the development of autoim- mune diseases such as RA or SpA. Some evidence reported that MSCs from patients with RA display impaired function in inhibiting Th17 cells and the same cells display defects in several important biological activities, especially the capacity to inhibit Th17 cell polarization. These functionally impaired MSCs may contribute to the development of arthritis (33). Further indirect evidence of MSCs involvement in the pathogenesis of SpA comes from some studies on psoriasis.

Liu et al. showed that BM-MSCs of patients with psoriasis exhibit abnormal cytokine secretion and furthermore, MSCs in psoriatic skin lesions were weaker inhibitors of T-cell proliferation.

This evidence could suggest that the attenuated inhibitory effect on T-cell proliferation might be one of the pathogenic mechanisms of psoriasis (34). Nevertheless, MSCs obtained from AS patients were shown to demonstrate normal proliferation, cell viability, surface markers and multiple differentiation characteristics and their immunomodulatory potential were significantly reduced when compared to that of MSCs from healthy donors. This evidence proved that the reduced immunomodulatory potential of MSCs played a crucial role in the pathogenesis of AS (35).

\section{MSCS AT ENTHESEAL SITES IN SpAS: ROLE AND FUNCTION}

In SpAs, inflammation can be found both in synovium and enthesis: in these anatomical sites, MSCs are present and it has been hypothesized that resident MSCs may function as a reservoir of stem cells for the regeneration or repair of joint tissues such as the articular cartilage, which have limited repair potential (36). Experimental data confirm the higher speed and quality of lesion repair of mesenchymatous cell injection with respect to chondrocyte injection in a rat model of degenerative joint disease (37). Other studies evaluated the optimal conditions for the differentiation of MSCs to tenocytes, chondrocytes, or bone cells 
according to their position in the enthesis. The results showed that mesenchymatous cell differentiation toward a tendon or bone phenotype depended on the degree of tensile loading: higher tensile loads promoted osteogenic differentiation (38). Mechanosensitive calcium permeable channels, such as the TRP channel, generate changes in intracellular calcium concentration in response to various mechanical stimuli (39-54). Activation of these channels at the level of plasma membrane of MSCs would induce intracellular calcium release and confirm the hypothesis that the mechanical tension could also activate MSCs in the pathological process that leads to the development of the SpAs (55-57). The activation and differentiation of MSCs at entheseal sites is also strongly influenced by paracrine signals (including the collagen-1 or fibronectin concentration), at least in the absence of strong tensile loads. High fibronectin concentrations promote osteogenic differentiation, whereas high collagen-1 concentrations inhibit osteogenic differentiation and promote differentiation to tenocytes (38).

These findings could provide indirect evidence of the involvement of MSCs in the pathological process affecting the enthesis in SpAs. However, the precise mechanism linking the inflammatory process to osteoproliferation in SpA is still unknown. Other data derive from studies on osteoarthritis: human osteophytes have shown them to contain cells exhibiting a mesenchymatous cell phenotype and probably originating in the periosteum $(58,59)$.

It has been demonstrated that during the osteogenic differentiation an imbalance between bone morphogenic protein (BMP)-2 and Noggin induces abnormal osteogenic differentiation of MSCs in AS patients. This result could reveal a mechanism of pathological osteogenesis in SpA and provides a new perspective on inhibiting pathological osteogenesis by regulating the balance between the BMP 2 and Noggin, or by directly inhibiting MSCs function (60). On this basis it is possible to hypothesize a role for MSCs in the radiographic progression in SpA. However, to the best of our knowledge, no studies have been performed on entheseal MSCs in the pathogenesis of SpA.

\section{MSCS IN THE TREATMENT OF DEGENERATIVE AND INFLAMMATORY ARTHRITIS: POSSIBLE THERAPEUTIC STRATEGY IN SPAS}

The regenerative, immunosuppressive and anti-inflammatory properties of cultured MSCs led to the development of trials for their therapeutic potential in preclinical models of inflammatory arthritis. As discussed above, several studies suggested that bone marrow- or adipose-derived MSCs have the ability to reset the immune system by reducing the pro-inflammatory Th1/Th17 response and enhancing the protective regulatory $\mathrm{T}$ cell response (61). Bone marrow has been the historic source site of MSCs and is the best studied. Some authors have found a trophic advantage with BM-MSCs; however, newer evidence suggests that BM-derived MSCs may be less potent in comparison to MSCs that reside in adipose tissue and this could be due to the physiologic stress and high turnover present in bone marrow compared with adipose $(62,63)$. The primary intent of the use of MSCs was to exploit their regenerative capacity to repair cartilage or bone damage in both inflammatory or degenerating pathologies such as RA or osteoarthritis (64). In this context, several studies on animal models of osteoarthritis have been performed with alternating results because of the number of variables. Moreover, there are other aspects still to be considered before translating this approach into clinical practice. The first issue to consider is donor source. Autologous products may be safer and almost free from immunogenicity, but in different studies, allogenic MSCs were used. Although MSCs are immuno-privileged with low levels of MHC-molecules, allogenic MSC have been shown to be immunogenic and are immune rejected by MHC class I- and class II-mismatched recipient mice (65). No clear indications have been determined, but autologous products 
are more common, probably due to fewer regulatory issues, even when injected directly into a joint. The second issue to consider is the possibility that injected MSCs could spread to other tissues. However, recent research demonstrates that MSCs injected in a joint remain localized to the transplant site without mobilization to distant organs (66). Another important issue to consider when administrating MSCs was the safety of transplantation and/or infusion/injection. Evidence is scarce; however, MSCs treatment could be considered safe. One study suggested that neither tumors nor infections were observed in a mean of 75 months after autologous BM-MSCs transplantation among 45 large joints in 41 patients (67). To exploit their immune-modulatory properties, MSCs can be directly infused in the blood stream, alongside their action as local regenerative factors. Two large studies on RA patients have been published: in the first, 136 patients with active RA who had inadequate responses to traditional medication received $40 \times 10^{6}$ allogeneic umbilical-cordderived MSC (UC-MSC) and 36 patients only received the cell-solvent without the cells (68). Patients were divided into two groups for different treatment: one group was given disease-modifying anti-rheumatic drugs (DMARDs) without UC-MSC, the other was given DMARDs plus UC-MSC via intravenous injection. The serum levels of TNF and IL-6 decreased after the first UC-MSC treatment, while the percentage of regulatory $\mathrm{T}$ cells of peripheral blood was increased. The treatment induced a significant improvement of disease activity and quality of life. The therapeutic effects were maintained for 3 and 6 months without continuous administration, correlating with the increased percentage of regulatory $\mathrm{T}$ cells of peripheral blood. In comparison, there were no such benefits observed in the control group of DMARDs without UCMSC. No patients showed acute serious side-effects either during or after UC-MSC infusion, and $4 \%$ showed mild adverse effects during the infusion, such as chill and/or fever, which disappeared within 2 $\mathrm{h}$ without any treatment and the authors reported that no major abnormal findings in hematologic or serum chemical profiles were found in the study. Thus, these data indicate that treatment with DMARDs plus UC-MSC may provide safe, significant, and persistent clinical benefits for patients with active RA (68). The second study presented at the American College of Rheumatology in San Diego in 2013 showed that in 53 refractory RA patients, allogeneic adipose-derived MSC infusion was safe at high dose (69). Dose-limiting safety signals were not identified and only one of the 53 patients experienced a serious adverse event leading to discontinuation of the treatment (lacunar infarction). Other adverse events were mild and transient (69). In this context, Wang et al. recently published the first clinical trial on the efficacy and safety of human MSCs in the treatment of AS.

The aim of the study was to assess the antiinflammatory properties of MSCs rather than their ability to repair tissue damage. In this study, patients with active disease received four intravenous infusions of allogenic MSCs on days 0, 7, 14, and 21. The results showed that the percentage of ASAS20 responders at the fourth week was $77.4 \%$ (24/31). Furthermore, in this study the authors evaluated the effects of MSCs infusion on the inflammatory lesions at MRI. The average total inflammation extent detected by MRI decreased from baseline to $4^{\text {th }}$ week $(p>0.05)$ and to $20^{\text {th }}$ week $(\mathrm{p}<0.05)$. Infusion of MSCs was well tolerated and no adverse events were reported. The authors stated that intravenous infusion of MSCs is a feasible, safe, and promising treatment for patients with AS. However, this study had some limitations such as the lack of a control group, the small number of enrolled patients and the lack of assessment of radiographic progression (70).

\section{CONCLUSIONS}

In conclusion, there is increasing evidence of the efficacy and safety of stem cells therapy in SpA or other types of autoimmune diseases. In the past, different case reports 
were published on the usage of hematopoietic stem cells transplantation in cases of $\mathrm{SpA}$ associated with leukemia, with encouraging results. However, there were complications of hematopoietic stem cells transplantation in the treatment of autoimmune diseases, which included availability, treatment-related mortality, infective and endocrinological complications, treatmentassociated toxicity, and secondary autoimmune diseases and malignancies $(71,72)$. MSCs, on the contrary, could represent a future treatment, with a possible safe and tolerable profile, for inflammatory arthritis such as SpAs. However, several questions remains about their use in humans: syngeneic or allogeneic cells, the tissue of origin of MSCs, timing of treatment, the number of cells injected, the route of injection, treatment regimes and different culture conditions. Potential long-term side effects as well as ethical considerations should be taken into account and further studies are needed.

Conflict of interest: the authors declare that there are no conflicts of interest.

\section{REFERENCES}

1. Moll JM, Haslock I, Macrae IF, et al. Associations between ankylosing spondylitis, psoriatic arthritis, Reiter's disease, the intestinal arthropathies, and BehCet's syndrome. Medicine (Baltimore). 1974; 53: 343-64.

2. Rudwaleit M. New approaches to diagnosis and classification of axial and peripheral spondyloarthritis. Curr Opin Rheumatol. 2010; 22 . 375-80.

3. Brown MA. Breakthroughs in genetic studies of ankylosing spondylitis. Rheumatology (Oxford). 2008; 47: 132-7.

4. Veale DJ. The epidemiology of psoriatic arthritis: fact or fiction? J Rheumatol. 2000; 27: 1105-6.

5. Braun J, Sieper J. Ankylosing spondylitis. Lancet. 2007; 369: 1379-90.

6. Callhoff J, Sieper J, Weiß A, et al. Efficacy of TNF- $\alpha$ blockers in patients with ankylosing spondylitis and non-radiographic axial spondyloarthritis: a meta-analysis. Ann Rheum Dis. 2015; 74: 1241-8.

7. Baeten D, Baraliakos X, Braun J, et al. Anti-interleukin-17A monoclonal antibody secukinumab in treatment of ankylosing spondylitis: a randomised, double-blind, placebocontrolled trial. Lancet. 2013; 382: 1705-13.
8. Poddubnyy D, Hermann KG, Callhoff J, et al. Ustekinumab for the treatment of patients with active ankylosing spondylitis: results of a 28-week, prospective, open-label, proofof-concept study (TOPAS). Ann Rheum Dis. 2014; 73: 817-23.

9. Spadaro A, Lubrano E, Marchesoni A, et al. Remission in ankylosing spondylitis treated with anti-TNF- $\alpha$ drugs: a national multicentre study. Rheumatology (Oxford). 2013; 52: 1914-9.

10. Perrotta FM, Marchesoni A, Lubrano E. Minimal disease activity and remission in psoriatic arthritis patients treated with anti-TNF- $\alpha$ Drugs. J Rheumatol. 2016; 43: 350-5.

11. Glenn JD, Whartenby KA. Mesenchymal stem cells: Emerging mechanisms of immunomodulation and therapy. World J Stem Cells. 2014; 6: 526-39.

12. Dominici M, Le Blanc K, Mueller I, et al. Minimal criteria for defining multipotent mesenchymal stromal cells. The International Society for Cellular Therapy position statement. Cytotherapy. 2006; 8: 315-7.

13. Chang H, Docheva D, Knothe UR, et al. Arthritic periosteal tissue from joint replacement surgery: a novel, autologous source of stem cells. Stem Cells Transl Med. 2014; 3: 308-17.

14. Koga H, Muneta T, Ju YJ, et al. Synovial stem cells are regionally specified according to local microenvironments after implantation for cartilage regeneration. Stem Cells. 2007; 25: 689-96.

15. Sakaguchi Y, Sekiya I, Yagishita K, et al. Comparison of human stem cells derived from various mesenchymal tissues e superiority of synovium as a cell source. Arthritis Rheum. 2005; 52: 2521-9.

16. Kolf CM, Cho E, Tuan RS. Mesenchymal stromal cells. Biology of adult mesenchymal stem cells: regulation of niche, self-renewal and differentiation. Arthritis Res Ther. 2007; 9: 204.

17. Caplan AI, Dennis JE. Mesenchymal stem cells as trophic mediators. J Cell Biochem. 2006; 98: 1076-84.

18. Glenn JD, Whartenby KA. Mesenchymal stem cells: emerging mechanisms of immunomodulation and therapy. World J Stem Cells. 2014; 6: 526-39.

19. De Bari C. Are mesenchymal stem cells in rheumatoid arthritis the good or bad guys? Arthritis Res Ther. 2015; 1: 113.

20. Jones E, Churchman SM, English A, et al. Mesenchymal stem cells in rheumatoid synovium: enumeration and functional assessment in relation to synovial inflammation level. Ann Rheum Dis. 2010; 69: 450-7.

21. Jones EA, English A, Henshaw K, et al. Enumeration and phenotypic characterization of synovial fluid multipotential mesenchymal 
progenitor cells in inflammatory and degenerative arthritis. Arthritis Rheum. 2004; 50: 817-27.

22. Suzawa M, Takada I, Yanagisawa J, et al. Cytokines suppress adipogenesis and PPARgamma function through the TAK1/TAB1/ NIK cascade. Nat Cell Biol. 2003; 5: 224-30.

23. Lories RJ, Derese I, De Bari C, et al. Evidence for uncoupling of inflammation and joint remodeling in a mouse model of spondylarthritis. Arthritis Rheum. 2007; 56: 489-97.

24. Ren G, Zhang L, Zhao X, et al. Mesenchymal stem cell-mediated immunosuppression occurs via concerted action of chemokines and nitric oxide. Cell Stem Cell. 2008; 2: 141-50.

25. Dazzi F, Krampera M. Mesenchymal stem cells and autoimmune diseases. Best Pract Res Clin Haematol. 2011; 24: 49-57.

26. Schett G, Elewaut D, McInnes IB, et al. How cytokine networks fuel inflammation: toward a cytokine-based disease taxonomy. Nat Med. 2013; 19: 822-4.

27. Di Ianni M, Del Papa B, De Ioanni M, et al. Mesenchymal cells recruit and regulate $\mathrm{T}$ regulatory cells. Exp Hematol. 2008; 36: 309-18.

28. Ghannam S, Pene J, Moquet-Torcy G, et al. Mesenchymal stem cells inhibit human Th17 cell differentiation and function and induce a T regulatory cell phenotype. J Immunol. 2010; 185: 302-12.

29. Djouad F, Charbonnier LM, Bouffi C, et al. Mesenchymal stem cells inhibit the differentiation of dendritic cells through an interleukin6-dependent mechanism. Stem Cells. 2007; 25: 2025-32.

30. Jiang XX, Zhang Y, Liu B, et al. Human mesenchymal stem cells inhibit differentiation and function of monocyte derived dendritic cells. Blood. 2005; 105: 4120-6.

31. Aggarwal S, Pittenger MF. Human mesenchymal stem cells modulate allogeneic immune cell responses. Blood. 2005; 105: 1815-22.

32. Djouad F, Bony C, Haupl T, et al. Transcriptional profiles discriminate bone marrowderived and synovium-derived mesenchymal stem cells. Arthritis Res Ther. 2005; 7: 130415.

33. Sun Y, Deng W, Geng L, et al. Mesenchymal stem cells from patients with rheumatoid arthritis display impaired function in inhibiting Th17 cells. J Immunol Res. 2015 [Epub ahead of print].

34. Liu R, Wang Y, Zhao X, et al. Lymphocyte inhibition is compromised in mesenchymal stem cells from psoriatic skin. Eur J Dermatol. 2014; 24: 560-7.

35. Wu Y, Ren M, Yang R, et al. Reduced immunomodulation potential of bone marrowderived mesenchymal stem cells induced CCR4+CCR6+Th/Treg cell subset imbalance in ankylosing spondylitis. Arthritis Res Ther. 2011; 13: R29.
36. De Bari C, Kurth TB, Augello A. Mesenchymal stem cells from development to postnatal joint homeostasis, aging, and disease. Birth Defects Res C Embryo Today. 2010; 90: 257-71.

37. Nourissat G, Diop A, Maurel N, et al. Mesenchymal stem cell therapy regenerates the native bone-tendon junction after surgical repair in a degenerative rat model. PLoS One. 2010; 5: e12248.

38. Rui YF, Lui PP, Ni M, et al. Mechanical loading increased BMP-2 expression which promoted osteogenic differentiation of tendon-derived stem cells. J Orthop Res. 2011; 29: 390-6.

39. Clapham DE. Calcium signaling. Cell. 2007; 131: 1047-58.

40. Orrenius S, Zhivotovsky B, Nicotera P. Regulation of cell death: the calcium-apoptosis link. Nat Rev Mol Cell Biol. 2003; 4: 552-65.

41. West AE, Chen WG, Dalva MB, et al. Calcium regulation of neuronal gene expression. Proc Natl Acad Sci USA. 2001; 98: 11024-31.

42. Pillozzi S, Becchetti A. Ion channels in hematopoietic and mesenchymal stem cells. Stem Cells Intern. 2012; 217910.

43. Berridge MJ, Bootman MD, Roderick HL, et al. Calcium signalling: dynamics, homeostasis and remodeling. Nat Rev Mol Cell Biol. 2003; 4: 517-29.

44. Moccia F, Lodola F, Dragoni S, et al. Ca2+ signalling in endothelial progenitor cells: a novel means to improve cell-based therapy and impair tumour vascularization. Curr Vasc Pharmacol. 2014; 12: 87-105.

45. Berridge MJ. The endoplasmic reticulum: a multifunctional signaling organelle. Cell Calcium. 2002; 32: 235-49.

46. Moccia F, Guerra G. Ca2+ Signalling in endothelial progenitor cells: friend or foe?. J Cell Physiol. 2016; 231: 314-27.

47. Moccia F, Zuccolo E, Soda T, et al. Stim and Orai proteins in neuronal $\mathrm{Ca}(2+)$ signaling and excitability. Front Cell Neurosci. 2015; 24: 153.

48. Kim TJ, Seong J, Ouyang M, et al. Substrate rigidity regulates $\mathrm{Ca} 2+$ oscillation via RhoA pathway in stem cells. J Cell Physiol. 2009; 218: 285-93.

49. Kawano S, Shoji S, Ichinose S, et al. Characterization of $\mathrm{Ca} 2+$ signaling pathways in human mesenchymal stem cells. Cell Calcium. 2002; 32: 165-74.

50. Sun S, Liu Y, Lipsky S, et al. Physical manipulation of calcium oscillations facilitates osteodifferentiation of human mesenchymal stem cells. FASEB J. 2007; 21: 1472-80.

51. Eijkelkamp N, Quick K, Wood JN. Transient receptor potential channels and mechanosensation. Annu Rev Neurosci. 2013; 36: 519-46.

52. Gees M, Owsianik G, Nilius B, et al. TRP channels. Compr Physiol. 2012; 2: 563-608.

53. Patel A, Sharif-Naeini R, Folgering JR, et al. Canonical TRP channels and mechanotrans- 
duction: from physiology to disease states. Pflugers Arch. 2010; 460: 571-81.

54. Ronco V, Potenza DM, Denti F, et al. A novel $\mathrm{Ca} 2+-$ mediated cross-talk between endoplasmic reticulum and acidic organelles: Implications for NAADP-dependent Ca2+ signaling. Cell Calcium. 2015; 57: 89-100.

55. Kim TJ, Sun J, Lu S, et al. Prolonged mechanical stretch initiates intracellular calcium oscillations in human mesenchymal stem cells. PLoS One. 2014; 9: e109378.

56. Kim TJ, Joo C, Seong J, et al. Distinct mechanisms regulating mechanical force-induced $\mathrm{Ca}^{2+}$ signals at the plasma membrane and the ER in human MSCs. Elife. 2015; 4: e04876.

57. Liu D, Yi C, Wang K, et al. Reorganization of cytoskeleton and transient activation of $\mathrm{Ca} 2+$ channels in mesenchymal stem cells cultured on silicon nanowire arrays. ACS Appl Mater Interfaces. 2013; 5: 13295-304.

58. Sharma RI, Snedeker JG. Paracrine interactions between mesenchymal stem cells affect substrate driven differentiation toward tendon and bone phenotypes. PLoS One. 2012; 7: e31504.

59. Berthelot JM, Le Goff B, Maugars Y. Pathogenesis of hyperostosis: a key role for mesenchymatous cells? Joint Bone Spine. 2013; 80: 592-6.

60. Xie Z, Wang P, Li Y, et al. Imbalance between BMP2 and Noggin induces abnormal osteogenic differentiation of mesenchymal stem cells in ankylosing spondylitis. Arthritis Rheumatol. 2016; 68: 430-40.

61. Swart JF, Wulffraat NM. Mesenchymal stromal cells for treatment of arthritis. Best Pract Res Clin Rheumatol. 2014; 28: 589-603.

62. Wyles CC, Houdek MT, Behfar A, et al. Mesenchymal stem cell therapy for osteoarthritis: current perspectives. Stem Cells Cloning. 2015; 8: 117-24.

63. Caplan AI. Cell delivery and tissue regeneration. J Control Release. 1990; 11: 157-65.
64. Kristjansson B, Honsawek S. Current perspectives in mesenchymal stem cell therapies for osteoarthritis. Stem Cells Intern. 2014: 194318.

65. Eliopoulos N, Stagg J, Lejeune L, et al. Allogeneic marrow stromal cells are immune rejected by MHC class I- and class II mismatched recipient mice. Blood. 2005; 106: 4057-65.

66. Horie M, Sekiya I, Muneta T, et al. Intra-articular injected synovial stem cells differentiate into meniscal cells directly and promote meniscal regeneration without mobilization to distant organs in rat massive meniscal defect. Stem Cells. 2009; 27: 878-87.

67. Wakitani S, Okabe T, Horibe T, et al. Safety of autologous bone marrow-derived mesenchymal stem cell transplantation for cartilage repair in 41 patients with 45 joints followed for up to 11 years and 5 months. J Tissue Eng Regen Med. 2011; 5: 146-50.

68. Wang L, Wang L, Cong X, et al. Human umbilical cord mesenchymal stem cell therapy for patients with active rheumatoid arthritis: safety and efficacy. Stem Cells Dev. 2013; 22: 3192-202.

69. Alvaro-Gracia JM, Jover JA, Garcia-Vicuna $\mathrm{R}$, et al. Phase Ib/IIa study on intravenous administration of expanded allogeneic adiposederived mesenchymal stem cells in refractory rheumatoid arthritis patients. In: Abstract at the Annual Scientific Meeting ACR, 2013.

70. Wang P, Li Y, Huang L, et al. Effects and safety of allogenic mesenchymal stem cell intravenous infusion in active ankylosing spondylitis patients who failed NSAIDs: a 20-week clinical trial. Cell Transplant. 2014; 23: 1293-303.

71. Daikeler T, Tichelli A, Passweg J. Complications of autologous hematopoietic stem cell transplantation for patients with autoimmune diseases. Pediatr Res. 2012; 71: 439-44.

72. Wong RS. Role of stem cells in spondyloarthritis: pathogenesis, treatment and complications. Hum Immunol. 2015; 76: 781-8. 